\title{
Mineração
}

\section{O problema de amostragem manual na indústria mineral}

\author{
Alexandre Grigorieff \\ Engenheiro de Minas, MSc, Copelmi Mineração Ltda \\ E-mail:alex@copelmi.com.br \\ Joao Felipe C.L. Costa \\ Engenheiro de Minas, PhD, Professor do Departamento de Minas/UFRGS \\ E-mail:jfelipe@ufrgs.br \\ Jair Koppe \\ Engenheiro de Minas, PhD, Professor do Departamento de Minas/UFRGS \\ E-mail:jkoppe@ufrgs.br
}

\section{Resumo}

São freqüentes as diferenças informadas nas análises de parâmetros físico-químicos obtidas por laboratórios do produtor e do consumidor. Contratos comerciais tendem a penalizar o produtor se contaminantes do minério excederem limites preestabelecidos. Diferenças em análises laboratoriais são devidas a diversas fontes de erro, entre elas a amostragem, a preparação e a análise. A teoria da amostragem provém os meios para verificar a precisão e acuracidade de um dado protocolo amostral aplicado a minério desagregado (não in situ). Um procedimento para medição do erro em cada etapa do processo amostral é introduzido e os resultados são comparados ao erro calculado tendo como base a teoria de Gy. Os resultados demonstram a aplicabilidade do método e sua relevância para auditar um protocolo amostral qualquer. A metodologia é ilustrada em um estudo de caso na Mina do Recreio, RS, Brasil.

Palavras-chave: amostragem, erros, precisão, protocolo.

\begin{abstract}
Discrepancies in raw materials properties informed by both producer and consumer are a common problem in most areas of the industry. Commercial contracts in the mineral industry normally penalize the producer, if contaminants in the ore exceed established limits. Differences in lab analysis are due many sources of error, such as distinct practices used in two laboratories for sampling, preparation or analysis. Sampling theory provides the tools to analyze errors involved in sampling, preparation and analysis. This paper proposes a methodology to check the precision and accuracy of a given sampling protocol emphasizing the application of Gy's sampling theory to broken ore. The results show the applicability of the method and its relevance to audit sampling procedures aiming at error minimization. The methodology is illustrated in a case study at a major coal producer in Brazil.
\end{abstract}

Keywords: sampling, error, precision, protocol.

Artigo recebido em 15/07/2002 e aprovado em 06/09/2002. 


\section{Introdução}

Nos dias de hoje, a necessidade de fabricação de produtos com qualidade assegurada faz com que os procedimentos e equipamentos empregados para a realização do controle de qualidade cada vez tenham que apresentar resultados mais confiáveis. Assim, uma boa estimativa do erro a ser gerado por um sistema de controle de qualidade tornou-se fundamental para possibilitar que o seu dimensionamento garanta a precisão e acuracidade exigidas. É preciso que os resultados do controle de qualidade não apresentem erros tendenciosos significativos, assim como o erro (aleatório) não deve ter uma variabilidade maior que a aceitável pelas partes interessadas.

No caso da indústria mineral, o controle de qualidade pode ser dividido em três etapas:

i. Amostragem ou seleção da amostra.

ii. Preparação da amostra.

iii. Análise da amostra preparada.

$\mathrm{O}$ alicerce de um bom programa de controle de qualidade está baseado na execução correta da operação de amostragem. Isto é obtido através do estabelecimento de um procedimento de amostragem que seja tanto acurado quanto preciso. Embora as técnicas de amostragem utilizadas na indústria mineral continuem a melhorar, a amostragem ainda é uma área muitas vezes negligenciada. As avaliações de qualidade dos produtos gerados muitas vezes são baseadas em amostras com sérios erros sistemáticos (tendenciosos) e com grandes variâncias de erro, provocando problemas para a aceitação dos lotes ou para a economicidade dos processos onde estes produtos minerais serão usados (Magri and Ortiz, 2000).

A representatividade de uma amostra ocorre quando existe a combinação da acuracidade e da reprodutibilidade. A acuracidade pode ser entendida como sendo a minimização do erro sistemático da amostragem. A reprodutibilidade ou precisão é a medida da dispersão dos resultados de qualidade obtidos para um mesmo lote (Mwasinga, 2000).

De acordo com Gy (1998), a média do erro e a variância do erro nunca são nulas estruturalmente. A representatividade de uma amostra ocorre quando a combinação desses dois fatores apresenta valores menores que o máximo estabelecido entre as partes interessadas, usualmente produtor e consumidor.

Ainda, segundo a teoria da amostragem de Gy (1982), existem alguns erros de amostragem que não podem ser eliminados: erro fundamental, erro de segregação e grupamento e erro de integração. Como conseqüência, sempre existirá um erro associado ao resultado de qualidade de um lote. Como o resultado de qualidade de um lote nunca é um valor exato, interpreta-se que esse resultado apresenta um comportamento de natureza estocástica. Dessa forma, é possível estimar, para qualquer resultado de controle de qualidade, uma faixa de erro devida a fatores relacionados à variabilidade existente entre as partículas do material amostrado.

Infelizmente, esse comportamento não é levado em consideração nem pelos contratos de comercialização de carvão e nem pelas Normas Técnicas de Amostragem, ocasionando freqüentes desentendimentos entre as partes e suscitando dúvidas sobre a qualidade dos trabalhos executados nos laboratórios. Além disso, uma das regras básicas da teoria da amostragem continua sendo desconsiderada por muitas Normas Técnicas (ISO 1998a e 1998b; ASTM 1996). Essa regra prega que qualquer partícula componente do lote deve possuir a mesma probabilidade de ser coletada e pertencer a amostra que servirá para a realização do controle de qualidade. A amostragem que segue essa regra é considerada estatisticamente correta. Dessa forma, é possível estimar, para qualquer resultado de controle de qualidade, uma faixa de erro devida a fatores relacionados à variabilidade do material particulado. Os tipos de amostragem considerados estatisticamente corretos, desde que seguidos alguns cuidados de dimensionamento e operação, são os executados por augers e por amostradores automáticos instalados em transportadores de correia. Todos os outros tipos de amostragem, inclusive a amostragem manual, são considerados incorretos.
No Brasil, a amostragem manual ainda é empregada por muitas empresas. Isto motivou a realização desse trabalho, o qual tem por finalidade investigar e quantificar o erro associado com as práticas de amostragem manual comumente utilizadas na indústria mineral.

\section{Metodologia}

A amostragem manual em pilha (Figura 1) é efetuada através do uso de uma pá, sendo coletadas as partículas que se encontram posicionadas nas porções mais superficiais da pilha. A inconformidade dessa prática de amostragem com a teoria da amostragem reside no fato de que a mesma é não-probabilística, pois pressupõe que as partículas dispostas nas porções inferiores da pilha e, portanto, inalcançáveis pela pá, apresentem exatamente as mesmas características de qualidade das partículas posicionadas na superfície.

Para verificar a possibilidade dos erros provocados pela amostragem manual serem passíveis de estimação e ficarem restritos à faixa de erro calculada pela teoria da amostragem, foi empregado o comparativo da faixa de erro dos teores de cinzas calculada pela teoria da amostragem (Gy, 1998, p. 71) com as diferenças obtidas da análise do lote de carvão em duas posições distintas do depósito.

Os ensaios foram realizados empregando a estrutura do laboratório de controle de qualidade da Mina do Recreio pertencente a Copelmi Mineração Ltda, localizado em Butiá no sul do Brasil. Todos os carvões utilizados foram oriundos do processo de lavagem sendo formados seis lotes de 250 tcada (Grigorieff, 2002).

Cada lote serviu para a execução de um ensaio. Cada ensaio foi estruturado seguindo os seguintes passos:

1. O lote, depositado na posição 1 , foi amostrado através do emprego de uma pá, sendo tomados 85 incrementos com aproximadamente $3 \mathrm{~kg}$ cada um, totalizando um peso de amostra de $250 \mathrm{~kg}$.

2. A operação de amostragem do lote depositado na posição 1 foi repetida 
três vezes. Em cada uma das vezes, a amostra foi colhida por um empregado diferente.

3. Cada amostra coletada foi preparada e analisada seguindo exatamente o mesmo procedimento. $\mathrm{O}$ teor de cinzas foi o parâmetro de qualidade analisado, sendo efetuadas duas determinações para cada amostra.

4. A seguir o carvão constituinte do lote foi transportado para outro local do depósito, sendo depositado na posição 2. A mudança de posição do lote provocou um rearranjo na disposição das partículas no interior do lote.

5. Os passos 1, 2 e 3 foram repetidos para o lote depositado na posição 2 .

Essa operação simulou a situação que usualmente ocorre na indústria carbonífera brasileira, onde o carvão é amostrado pelo produtor (posição 1) e depois é amostrado pelo consumidor (posição 2).

\section{Comparativo da faixa de erro dos teores de cinzas calculada pela teoria da amostragem com as diferenças obtidas pela mudança de posição de depósito do lote}

O tratamento dessa questão foi dividido em três partes. Inicialmente foi efetuada a determinação dos valores das variâncias de erro definidas pela teoria da amostragem, seu desvio-padrão e o intervalo de erro esperado, considerando uma confiabilidade de $95 \%$. A seguir foi realizada a determinação das diferenças encontradas entre os valores de cinzas encontrados para a posição 1 e para a posição 2 (Tabela 1). Para avaliar as diferenças entre posições (1 e 2) apresentadas na Tabela 1, utilizou-se o erro fundamental (Gy, 1982) definido por:

$s^{2} E F=\left(1 / M_{E}-1 / M_{L}\right) \cdot C \cdot d^{3}$

No caso: $M_{E}$ é a massa de amostra (g) em um dado estágio do processo de amostragem, $\mathrm{M}_{\mathrm{L}}$ é a massa do lote ( $\mathrm{g}$ ) ou massa inicial da etapa de preparação, C é a constante de amostragem de um determinado minério e dé o top size das partículas da amostra $(\mathrm{cm})$.

A constante de amostragem $\mathrm{C}$ é obtida através da seguinte fórmula:

$\mathrm{C}=\mathrm{c} .1 . \mathrm{f} . \mathrm{g}$

No caso: 1 lé o fator de liberação $\left(1=\mathrm{d}_{\mathrm{lib}} / \mathrm{d}\right)^{0.5}$, $\mathrm{d}_{\text {lib }}$ é o tamanho de liberação do minério analisado, f é o fator de forma das partículas, usualmente utiliza-se 0,5 ; $\mathrm{g}$ é o fator da faixa de tamanho e c é o fator de composição mineralógica, dado por:

$c=(1-a)\left[(1-a) \rho_{1}+a \rho_{2}\right] / a$

No caso: a é a concentração do componente do minério em consideração (adimensional), $\rho_{1}$ é a densidade das partículas do componente crítico $\left(\mathrm{g} / \mathrm{cm}^{3}\right), \rho_{2}$ é a densidade das partículas dos outros componentes do minério $\left(\mathrm{g} / \mathrm{cm}^{3}\right)$. $\mathrm{O}$ fator de faixa de tamanho g pode ser estimado a partir da relação entre o top size d e o limite de tamanho mais baixo d' (5\% de undersize), assim, para uma grande faixa de tamanhos $\left(\mathrm{d} / \mathrm{d}^{\prime}>4\right)$, o fator $\mathrm{g}$ é igual a 0,25 .

Além do erro fundamental, duas fontes adicionais de erro devem ser cal- culadas: (i) o erro de segregação e grupamento e (ii) o erro analítico. De acordo com Gy (1982), a variância de segregação e grupamento ( $\left.s^{2} E S G\right)$ é igual ou menor que a variância do erro fundamental ( $\left.\mathrm{s}^{2} \mathrm{EF}\right)$. Em consequência, é sempre seguro assumir que:

$s^{2} \mathrm{ESG}=\mathrm{s}^{2} \mathrm{EF}$

A variância do erro analítico $\left(\mathrm{s}^{2} \mathrm{EA}\right)$ se refere aos erros provocados pelos equipamentos e ao processo de análise da amostra moída. Para o caso do laboratório da Mina do Recreio, esse valor é igual a:

\section{$\mathrm{s}^{2} \mathrm{EA}=1,75 \times 10^{-7}$}

A Tabela 2 mostra os cálculos efetuados para a determinação das variâncias de erro do lote número 1. A variância do erro fundamental foi calculada para a amostragem do carvão e para o processo de preparação da amostra. A preparação da amostra foi dividida em quatro etapas. As etapas foram definidas na medida em que ocorreu a redução de massa e a granulometria da amostra, conforme:

i. $250 \mathrm{~kg}$ extraídos de 250 t usando amostragem manual.

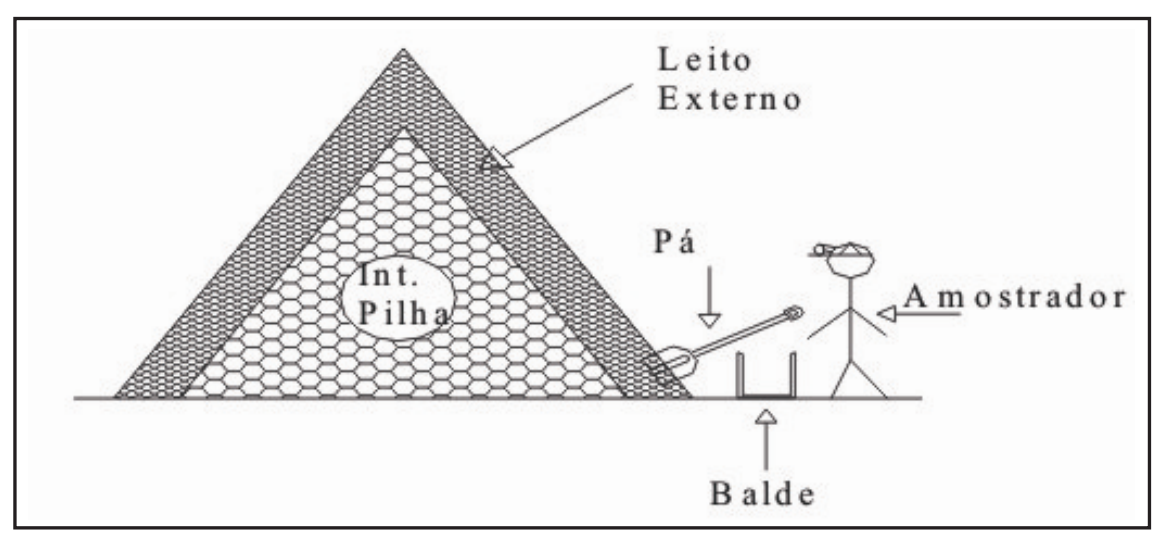

Figura 1 - Esquema utilizado na amostragem manual de pilhas de minério.

Tabela 1 - Conteúdo de cinzas determinado nas posições 1 e 2 por quatro diferentes amostradores. As amostras foram analisadas em duplicatas.

\begin{tabular}{c|c|c|c|c}
\hline \multirow{2}{*}{ Amostrador } & \multicolumn{2}{|c|}{ Cinza (\%) na Posição 1 } & \multicolumn{2}{c}{ Cinza (\%) na Posição 2 } \\
\cline { 2 - 5 } & Alíquota 1 & Alíquota 2 & Alíquota 1 & Alíquota 2 \\
\hline 1 & 32,93 & 32,74 & 31,38 & 31,96 \\
\hline 2 & 32,93 & 31,93 & 31,08 & 31,71 \\
\hline 3 & 31,41 & 32 & 31,88 & 31,85 \\
\hline 4 & 32,49 & 32,53 & 30,9 & 31,52 \\
\hline
\end{tabular}


ii. $40 \mathrm{~kg}$ extraídos de $250 \mathrm{~kg}$ depois de homogeneizar e quartear.

iii. $10 \mathrm{~kg}$ extraídos de $40 \mathrm{~kg}$ após britar, homogeneizar e quartear.

iv. 400 g extraídos de 10 kg após moer, homogeneizar e quartear.

v. 1 g usado para determinar o teor de cinzas extraído de 400 g após homogeneização.

O valor da variância do erro de segregação e grupamento foi igual ao da variância do erro fundamental, conforme a Equação (4).

A variância do erro de amostragem total é o somatório das variâncias indicadas na Tabela 2 acrescida da variância do erro de segregação e grupamento e da variância do erro analítico e é igual a 4,32 x $10^{-4}$. O desvio-padrão do erro de amostragem total é de 2,08 x 10-2. A variância e o desvio-padrão do erro total são valores relativos. Para determinar o intervalo de erro com $95 \%$ de confiabilidade, é necessário converter o desvio-padrão relativo para desvio-padrão absoluto, assim:

$(\mathrm{SE}$ Total $)$ absoluto $=(\mathrm{SE}$ Total $)$ relativo. $\%$ teor de cinzas

Para 95\% de confiabilidade, teremos:

Intervalo $= \pm 2 .($ SE Total $)$ absoluto $= \pm 1,35 \%$

Considerando que o carvão depositado na posição 1 se refere ao carvão amostrado pelo produtor, a teoria da amostragem para o protocolo empregado determina que o teor de cinzas a ser reproduzido pelo controle de qualidade do consumidor deveria estar inserido dentro do intervalo igual a:

$32,37 \% \pm 1,35 \%$, ou seja, entre $31,02 \%$ e $33,72 \%$ de cinzas.

As diferenças encontradas para o teor de cinzas entre as posições 1 e 2 do lote 1 foram calculadas. Os resultados são mostrados na Tabela 3 .

Observa-se que, no caso do lote 1 , cerca de $13 \%$ dos resultados ficaram fora do intervalo determinado pela teoria da amostragem $( \pm 1,35 \%)$. A seguir foi efetuado o mesmo ensaio com outros cinco lotes de carvão. Nesses ensaios, o percentual que

Tabela 2 - Variância do erro calculada para o lote 1.

\begin{tabular}{c|c|c|c|c|c}
\hline \multirow{2}{*}{ Parâmetro } & \multicolumn{5}{|c}{ Erro Fundamental (FE) em cada etapa de extração } \\
\cline { 2 - 6 } & Lote & Etapa 1 & Etapa 2 & Etapa 3 & Etapa 4 \\
\hline$M_{E}(\mathrm{~g})$ & $250 \times 10^{3}$ & $40 \times 10^{3}$ & 104 & 400 & 1 \\
\hline$M_{\mathrm{L}}(\mathrm{g})$ & $250 \times 10^{6}$ & $250 \times 10^{3}$ & $40 \times 10^{3}$ & 104 & 400 \\
\hline$\rho \mathrm{G}$ & 2,25 & 2,25 & 2,25 & 2,25 & 2,25 \\
\hline$\rho \mathrm{A}$ & 1,30 & 1,30 & 1,30 & 1,30 & 1,30 \\
\hline $\mathrm{A}$ & 0,3237 & 0,3237 & 0,3237 & 0,3237 & 0,3237 \\
\hline $\mathrm{c}\left(\mathrm{g} / \mathrm{cm}^{3}\right)$ & 4,06 & 4,06 & 4,06 & 4,06 & 4,06 \\
\hline $\mathrm{d}(\mathrm{cm})$ & 5,70 & 5,70 & 2,20 & 0,30 & 0,025 \\
\hline $\mathrm{d}_{\mathrm{lib}}(\mathrm{cm})$ & 0,025 & 0,025 & 0,025 & 0,025 & 0,025 \\
\hline $\mathrm{I}$ & 0,066 & 0,066 & 0,107 & 0,289 & 1,000 \\
\hline $\mathrm{f}$ & 0,50 & 0,50 & 0,50 & 0,50 & 0,50 \\
\hline $\mathrm{g}$ & 0,25 & 0,25 & 0,25 & 0,25 & 0,25 \\
\hline $\mathrm{C}\left(\mathrm{g} / \mathrm{cm}^{3}\right)$ & 0,0336 & 0,0336 & 0,0541 & 0,1464 & 0,5073 \\
\hline $\mathrm{s}^{2} \mathrm{FE}(\%)^{2}$ & $2,49 \times 10^{-5}$ & $1,31 \times 10^{-4}$ & $4,32 \times 10^{-5}$ & $9,49 \times 10^{-6}$ & $7,91 \times 10^{-6}$ \\
\hline
\end{tabular}

extrapolou o intervalo de erro calculado pela teoria da amostragem foi:

Lote 2 - 100\% das amostras não se enquadraram no intervalo.

Lote 3 - 44\% das amostras não se enquadraram no intervalo.

Lote 4 - 100\% das amostras não se enquadraram no intervalo.

Lote 5 - 44\% das amostras não se enquadraram no intervalo.

Lote 6 - 38\% das amostras não se enquadraram no intervalo.

\section{Discussão dos resultados}

O estudo realizado teve por finalidade simular a operação de controle de qualidade empregando a amostragem manual usualmente efetuada em muitas minas e consumidores de carvão brasileiros. O carvão amostrado na posição 1 teve a intenção de se referir à amostragem realizada no depósito da planta de beneficiamento, enquanto que o carvão da posição 2 representou a amostragem realizada pelo consumidor. As diferenças encontradas deveriam apresentar valores inferiores aos da faixa de erro estimada pela teoria da amostragem. Caso essa hipótese estivesse correta, poderia se concluir que a amostragem manual em pilha poderia ter seu erro previsto e, dessa forma, tornar-se uma operação confiável de controle de qualidade.

Foram realizados seis testes empregando carvões beneficiados com diferentes teores de cinzas. Os teores de cinzas dos lotes ensaiados variaram de $26,05 \%$ até $38,62 \%$. Todos os lotes apresentaram um peso de aproximadamente 250 toneladas. As operações de amostragem, preparação de amostras e análise observaram os procedimentos e cuidados indicados pelas normas técnicas brasileiras NBR 8289; NBR 8291 e NBR 8292. Cada lote foi amostrado quatro vezes na posição 1 e quatro vezes na posição 2 .

Os resultados do controle de qualidade de cada lote do carvão depositado na posição 1 foram comparados com os 
Tabela 3 - Diferenças em valor absoluto para o teor de cinzas considerando posição e amostrador.

\begin{tabular}{c|c|c|c|c|c|c|c|c}
\hline \multicolumn{3}{c|}{ Posição 1 } & \multicolumn{5}{c|}{ Posição 2 } & Diferença (\%) \\
\hline \multicolumn{3}{c}{ Amostrador } & \multicolumn{5}{c}{ Amostrador } & (\%) Cinzas \\
\hline $\mathrm{X}$ & 2 & 3 & 4 & 1 & 2 & 3 & 4 & \\
\hline $\mathrm{X}$ & & & & $\mathrm{X}$ & & & & 1,17 \\
\hline $\mathrm{X}$ & & & & & $\mathrm{X}$ & & & 1,44 \\
\hline $\mathrm{X}$ & & & & & & $\mathrm{X}$ & & 0,97 \\
\hline $\mathrm{X}$ & & & & & & & $\mathrm{X}$ & 1,63 \\
\hline & $\mathrm{X}$ & & & $\mathrm{X}$ & & & & 0,76 \\
\hline & $\mathrm{X}$ & & & & $\mathrm{X}$ & & & 1,03 \\
\hline & $\mathrm{X}$ & & & & & $\mathrm{X}$ & & 0,56 \\
\hline & $\mathrm{X}$ & & & & & & $\mathrm{X}$ & 1,22 \\
\hline & & $\mathrm{X}$ & & $\mathrm{X}$ & & & & 0,04 \\
\hline & & $\mathrm{X}$ & & & $\mathrm{X}$ & & & 0,31 \\
\hline & & $\mathrm{X}$ & & & & $\mathrm{X}$ & & 0,16 \\
\hline & & $\mathrm{X}$ & & & & & $\mathrm{X}$ & 0,50 \\
\hline & & & $\mathrm{X}$ & $\mathrm{X}$ & & & & 0,84 \\
\hline & & & $\mathrm{X}$ & & $\mathrm{X}$ & & & 1,11 \\
\hline & & & $\mathrm{X}$ & & & $\mathrm{X}$ & & 0,64 \\
\hline & & & $\mathrm{X}$ & & & & $\mathrm{X}$ & 1,30 \\
\hline
\end{tabular}

resultados do carvão depositado na posição 2. Foram obtidas 16 combinações de resultados, sendo efetuado o cálculo da diferença do teor de cinzas para cada combinação. Em todos os ensaios foram observadas diferenças significativas entre os resultados, da posição 1 e da posição 2. Foi verificado também que, para a maioria dos lotes, as diferenças de resultados existentes entre amostradores para o carvão situado na mesma posição não foram expressivas. Isto indica que as diferenças de teores de cinzas existentes tiveram uma maior influência da posição do carvão.

De acordo com a formulação da teoria da amostragem, as faixas de precisão calculadas deveriam conter, no mínimo, 95\% dos resultados. Em todos os seis ensaios, o percentual esteve sempre inferior a $95 \%$. Houve lotes em que todas as combinações de resultados tiveram um erro superior ao calculado pela teoria da amostragem. Em vários lotes, os resultados obtidos tiveram uma variação bastante superior ao esperado.

Torna-se claro que o teor de cinzas das partículas dispostas na porção superficial da posição 1 é diferente do teor das partículas dispostas na porção su- amostragem manual. Assim, nesse tipo de amostragem, além da variabilidade existente entre as partículas, os erros de amostragem são influenciados pela disposição das partículas no interior do lote. Como essa disposição é circunstancial, os resultados de qualidade são conseqüência das circunstâncias que envolvem a deposição do carvão. Em conseqüência, a precisão da amostragem manual não pode ser estimada, tornando-a não confiável. Conclui-se que a amostragem manual deve ser banida de qualquer sistema de controle de qualidade.

\section{Referências Bibliográficas}

GRIGORIEFF, A. Melhoria no controle de qualidade de na produção de carvão mineral com o uso da teoria de amostragem. Universidade Federal do Rio Grande do Sul, 2001. 178 p. (Dissertação de Mestrado).

GY, P.M. Sampling of particulate materials, theory and practice. Amsterdam: Elsevier, Second Revised Edition, 1982. 431 p.

GY, P.M. Sampling for analytical purposes. Chichester: England, John Wiley \& Sons Ltd, 1998. 153 p.

MWASINGA, P. P. Optimizing sampling protocols for a base metal deposit, Proceedings of Geostat 2000 (CD-ROM), KLEINGELD, W.J., KRIGE, D. (ed.) . 2000. p. 1-13.

MAGRI, E.V., ORTIZ, J.C. Estimation of economic losses due to poor blast hole sampling in open pits, In: Proceedings of Geostat 2000 (CD-ROM), 2000. p. 1-10.

ISO, 1998a. International Organization for Standardization, Draft International Standard ISO/DIS 13909-3, Hard Coal and Coke- Mechanical Sampling - Part 3: Coal - Sampling from Stationary Lots.

ISO, 1998b. International Organization for Standardization, Draft International Standard ISO/DIS 13909-4, Hard Coal and Coke - Mechanical Sampling - Part 4: Coal - Preparation of Test Samples.

A precisão da amostragem é variável porque depende da variabilidade existente entre as partículas do lote. A teoria da amostragem mede esta variabilidade e propicia o conhecimento do erro que se pode esperar para uma dada amostragem. No entanto, para que essa medição tenha efeito, é necessário que a amostragem seja efetuada corretamente, também sob o ponto de vista probabilístico.

Cada partícula componente do lote deve possuir a mesma probabilidade de pertencer ao lote. Este não é o caso da
ASTM, 1996. Annual Book of ASTM Standards, Gaseous Fuels; Coal and Coke, v. $05.05 .510 \mathrm{p}$.

ABNT, 1983. Associação Brasileira de Normas Técnicas, NBR 8291. Amostragem de Carvão Mineral Bruto e/ou Beneficiado.

ABNT, 1983b. Associação Brasileira de Normas Técnicas, NBR 8292. Preparação de Amostra de Carvão Mineral para Análise e Ensaios. Rio de Janeiro, Brasil. Rio de Janeiro, Brasil. 